\title{
Genome-wide identification and characterization of auxin response factor (ARF) family genes related to flower and fruit development in papaya (Carica papaya L.)
}

Kaidong Liu ${ }^{1,4^{*}}$, Changchun Yuan ${ }^{4}$, Haili Li $i^{4}$ Wanhuang Lin ${ }^{1}$, Yanjun Yang ${ }^{3}$, Chenjia Shen ${ }^{3^{*}}$ and Xiaolin Zheng ${ }^{2^{*}}$

\begin{abstract}
Background: Auxin and auxin signaling are involved in a series of developmental processes in plants. Auxin Response Factors (ARFs) is reported to modulate the expression of target genes by binding to auxin response elements (AuxREs) and influence the transcriptional activation of down-stream target genes. However, how ARF genes function in flower development and fruit ripening of papaya (Carica papaya L.) is largely unknown. In this study, a comprehensive characterization and expression profiling analysis of 11 C. papaya ARF (CpARF) genes was performed using the newly updated papaya reference genome data.

Results: We analyzed CPARF expression patterns at different developmental stages. CPARF1, CPARF2, CPARF4, CPARF5, and CPARF10 showed the highest expression at the initial stage of flower development, but decreased during the following developmental stages. CPARF6 expression increased during the developmental process and reached its peak level at the final stage of flower development. The expression of CPARF1 increased significantly during the fruit ripening stages. Many AuxREs were included in the promoters of two ethylene signaling genes (CPETR1 and CPETR2) and three ethylene-synthesis-related genes (CPACS1, CPACS2, and CPACO1), suggesting that CpARFs might be involved in fruit ripening via the regulation of ethylene signaling.

Conclusions: Our study provided comprehensive information on ARF family in papaya, including gene structures, chromosome locations, phylogenetic relationships, and expression patterns. The involvement of CPARF gene expression changes in flower and fruit development allowed us to understand the role of ARF-mediated auxin signaling in the maturation of reproductive organs in papaya.
\end{abstract}

Keywords: Auxin, Auxin response factor, Papaya, Developmental process, Fruit ripening

\footnotetext{
*Correspondence: liukaidong2001@126.com; shencj@hznu.edu.cn; zheng9393@163.com

${ }^{1}$ College of Bioscience and Technology, Hunan Agricultural University,

Changsha, Hunan 410128, China

${ }^{3}$ College of Life and Environmental Sciences, Hangzhou Normal University,

Hangzhou 310036, China

${ }^{2}$ College of Food Science and Biotechnology, Zhejiang Gongshang

University, Hangzhou 310035, China

Full list of author information is available at the end of the article
}

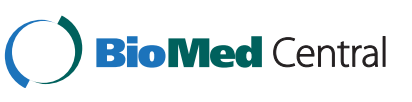

(c) 2015 Liu et al. Open Access This article is distributed under the terms of the Creative Commons Attribution 4.0 International License (http://creativecommons.org/licenses/by/4.0/), which permits unrestricted use, distribution, and reproduction in any medium, provided you give appropriate credit to the original author(s) and the source, provide a link to the Creative Commons license, and indicate if changes were made. The Creative Commons Public Domain Dedication waiver (http://creativecommons.org/publicdomain/zero/1.0/) applies to the data made available in this article, unless otherwise stated. 


\section{Background}

Auxin is a plant hormone that plays pivotal roles in the regulation of plant growth in response to diverse developmental and environmental events such as embryogenesis, organogenesis, tropic growth, root architecture, flower and fruit development, tissue and organ patterning, and vascular development [1-3]. It has been shown that auxin coordinates plant development essentially through the transcriptional regulation of some gene families, such as auxin/indole-3-acetic acid $(A u x / I A A)$, Gretchen Hagen3 (GH3), small auxin up RNA (SAUR), and auxin response factor $(A R F)[4,5]$. It was subsequently found that these so-called early auxin-responsive genes are characterized by conserved promoter elements, including the TGA element (AACGAC), core element of the auxin response region (AuxRE-core; GGTCCAT), and auxin response element (AuxRE; TGTCTC) [6, 7]. Being an important component of auxin signaling pathway, ARFs activate or repress the expression of auxin response genes by binding to AuxRE in their promoter [8].

A typical ARF contains a highly conserved $\mathrm{N}$-terminal B3-like DNA binding domain (DBD) that recognizes AuxRE in the promoter of auxin-responsive genes [8]. The C-terminal dimerization domain (CTD) contains two motifs, called III and IV, that are also found in Aux/IAA and enable the formation of homo- and hetero-dimers among ARFs and Aux/IAAs [9, 10]. The middle region (MR), located between DBD and CTD, confers transcriptional activation or repression depending on its amino acid composition $[8,11]$.

The functions of ARFs are well studied. In Arabidopsis thaliana, arf1 and arf2 loss-of-function mutations affect leaf senescence and floral organ abscission [12]. Lossof-function arf3 mutants display defects in gynoecium and floral meristem patterning $[13,14]$, while mutant arf5 is characterized by abnormal vascular strands and embryo axis [15]. AtARF7 is involved in the conditional regulation of differential growth in aerial tissues, and a mutation in AtARF7 impairs hypocotyl response to blue light and auxin stimuli [16]. AtARF8 regulates hypocotyl elongation, auxin homeostasis, and fruit development $[12,17]$. Furthermore, the flowers of arf6/arf8 double mutant are infertile closed buds with short petals, short stamen filaments, and undehisced anthers [18]. The double mutation, arf7/arf19 affects auxin mediated lateral root development [19]. In rice (Oryza sativa L.), transgenic plants that express an antisense OsARF1 show extremely low growth, poor vigor, curled leaves, and sterility, suggesting that this gene is essential for vegetative and reproductive development [20]. Previous studies have shown that OsARF16, a transcription factor regulating auxin redistribution, is required for iron and phosphate deficiency responses in rice [21-23]. Another auxin response factor, OsARF19, controls rice leaf angles through the positive regulation of OsGH3-5 and OsBRI1 [24]. In tomato (Solanum lycopersicon), recent studies have shown the involvement of SIARF genes in flower development and fruit set, development, and ripening [25-27].

Papaya (Carica papaya L.) is an economically important fruit crop in tropical and subtropical countries [28]. Sex type in this trioecious species is determined by a pair of sex chromosomes, and plants have either female $(\mathrm{XX})$, male $(\mathrm{XY})$, or hermaphrodite $[\mathrm{XY}(\mathrm{h})]$ flowers [29]. Papaya often exhibits male and imperfect hermaphrodite flowers, which are influenced by environmental and hormonal factors [30-32]. Under high summer temperatures, the flowers have been observed to change from hermaphrodite to male because of ovary abortion and stamen carpelloid. Some endohormones, such as auxin, may play important roles in this change process $[28,33]$. Despite the various causes of malformation in papaya fruit, the pear-shaped fruits from hermaphrodite flowers are commercially preferred, and hermaphrodite papayas are favored worldwide for economic production $[28,34]$. Papaya fruits are very susceptible to deterioration and postharvest losses mainly by fungal decay and physiological disorders such as chilling injury, pests, mechanical injury, and over-ripeness. Therefore, there are several critical problems in breeding and cultivation of hermaphrodite plants that need to be solved [35]. Auxin has a positive role in the quality maintenance and shelf life of harvested papaya fruits [36]. Application of exogenous auxin can delay fruit ripening in many crop species [34]; however, the underlying mechanism linking auxin signaling and reproduction of papaya is largely unknown.

As an important segment of the auxin-signaling pathway, ARFs are encoded by a multi-gene family in many different plant species. There are 23 members in Arabidopsis, 22 in tomato, 31 in maize (Zea mays L.), 15 in cucumber (Cucumis sativus), 39 in poplar (Populus trichocarpa), 25 in rice (Oryza sativa L.), 24 in Medicago (Medicago truncatula), 19 in sweet orange (Citrus sinensis), and 51 in soybean (Glycine max L.) [5, 7, 21, 37-42]. In this study, we used the existing data in public databases to perform domain analysis and identify genes encoding ARFs in papaya. We also aimed to reveal comprehensive information on the gene structure, protein motif architecture, and sequence homology of 11 CpARFs.

\section{Results}

Genome-wide identification of CPARF genes

A total of $11 A R F$ s were identified in C. papaya. These genes were named according to the phylogenetic relationships between C. papaya and Arabidopsis. Comprehensive information on these 11 CpARF genes, 
including gene name, locus ID, open reading frame (ORF) length, number of introns, location on supercontigs and deduced polypeptide sequences, is presented in Table 1. The size of deduced CpARFs ranged from 311 (CpARF6) to 938 amino acids (CpARF5), the corresponding molecular masses from 34.83 to $103.7 \mathrm{kDa}$, and the predicted isoelectric points from 5.16 (CpARF5) to 9.03 (CpARF6). All the nucleic acid sequences were listed in the Additional file 1: Table S1.

Analysis of phylogenetic relationships and gene structure The phylogenetic distribution suggested that $A R F \mathrm{~s}$ could be grouped into four major subclasses, including Ia, Ib, II, and III (Fig. 1a). Based on the phylogenetic tree, seven sister gene pairs were identified between Arabidopsis and C. papaya: CpARF2/AtARF2, CpARF3/AtARF3, CpARF4/ AtARF4, CpARF5/AtARF5, CpARF10/AtARF10, CpARF16/ AtARF16, and CpARF17/AtARF17. No sister gene pairs were found between $C$. papaya and rice. Most CpARFs contained three typical domains: DBD, domain II, and AUX/IAA family domain. CPARF2, CpARF3, CpARF6, and CpARF17 contained DBD and domain II, but no AUX/IAA family domain (Fig. 1b). The exon-intron structure of each $C p A R F$ was revealed by comparing the full-length cDNA sequences with the corresponding genomic DNA sequences. The number of introns in $C p A R F$ genes ranged from 1 to 13 (Fig. 1c). CpARF genes, even with close phylogenetic relationship, displayed complex distribution patterns of introns-exons.

\section{Analysis of amino-acid composition and classification of CPARFs}

The 11 CpARFs were classified into three groups based on their MR amino-acid composition and the presence or absence of CTDs: (1) ARFs with a DBD, activator MR and a CTD; (2) ARF with a DBD, repressor MR and a CTD; and (3) ARFs with a DBD, repressor MR, but no CTD (Fig. 2a and Additional file 2: Figure S1). The domain position in these 11 CPARFs is presented in Additional file 3: Table S2, and the amino acid composition of MRs is shown in Fig. 2b and Additional file 4: Table S3. CpARFs contained four putative transcriptional activators, CpARF5, seven, ten, and 16 (QSL-rich MR), and three putative transcriptional repressors, CPARF1, four, and 11 (SLPG-rich MR). Three CPARFs (CpARF2, three, and 17) were putative transcriptional repressors that did not contain a CTD. Only one CPARF, CpARF6, contained only a DBD.

\section{Expression patterns for CPARF genes in different plant tissues}

To study the physiological function of CpARF genes, the spatial-specific expression pattern of the 11 CpARF genes was detected in different tissues and organs, including shoots, leaves, flowers, fruits and roots. The expression of most CPARF genes was ubiquitous in all studied tissues and organs, suggesting that they might have a putative function in many aspects of plant growth and development. Some CpARF genes (CpARF2, CPARF6, CPARF10, CPARF16, and CPARF17) showed fruit-specific expression, which indicated that they might play a role in fruit ripening. CPARF1 was highly expressed in flowers, while CPARF3, CPARF5, and CPARF11 were highly expressed in roots. Many CpARF genes, including CPARF1, CpARF2, CpARF3, CpARF6, CpARF7, CpARF16, and $C P A R F 17$, were hardly detectable in leaves and shoots (Fig. 3).

Table 1 The information of ARF family genes in Carica papaya ${ }^{a}$

\begin{tabular}{|c|c|c|c|c|c|c|c|c|}
\hline \multirow[b]{2}{*}{ Gene ID } & \multirow[b]{2}{*}{ Name $^{b}$} & \multirow[b]{2}{*}{ Location $^{c}$} & \multirow[b]{2}{*}{ Direction } & \multirow[b]{2}{*}{ ORF length } & \multirow[b]{2}{*}{ Introns } & \multicolumn{3}{|c|}{ Deduced polypeptide } \\
\hline & & & & & & Length (aa) & Mol wt (kDa) & $\mathrm{pl}$ \\
\hline evm. TU.supercontig_9.161 & CpARF1 & supercontig_9:969763..974848 & Reverse & 2094 & 13 & 698 & 77.67 & 7.18 \\
\hline evm.TU.contig_31756.1 & CpARF2 & contig_31756:3939..7439 & Forward & 1855 & 11 & 619 & 68.86 & 7.12 \\
\hline evm.TU.supercontig_7.3 & CPARF3 & supercontig_7:132322..138926 & Reverse & 2022 & 10 & 674 & 73.19 & 7.01 \\
\hline evm.TU.supercontig_139.80 & CpARF4 & supercontig_139:638531..645762 & Reverse & 2439 & 11 & 813 & 89.78 & 6.58 \\
\hline evm.TU.supercontig_26.24 & CpARF5 & supercontig_26:231561..267729 & Reverse & 2814 & 13 & 938 & 103.7 & 5.16 \\
\hline evm.TU.supercontig_17.53 & CpARF6 & supercontig_17:617715..620541 & Reverse & 933 & 8 & 311 & 34.83 & 9.03 \\
\hline evm.TU.supercontig_261.2 & CPARF7 & supercontig_261:2520..11208 & Reverse & 2649 & 12 & 883 & 97.65 & 5.52 \\
\hline evm.TU.supercontig_65.4 & CpARF10 & supercontig_65:11160..14085 & Reverse & 1944 & 4 & 648 & 71.54 & 7.06 \\
\hline evm.TU.supercontig_96.40 & CpARF11 & supercontig_96:684489..688508 & Forward & 2064 & 13 & 688 & 76.05 & 6.66 \\
\hline evm.TU.supercontig_53.88 & CpARF16 & supercontig_53:584129..586644 & Reverse & 2091 & 2 & 697 & 76.94 & 6.57 \\
\hline evm.TU.supercontig_49.122 & CpARF17 & supercontig_49:862531..867248 & Reverse & 1809 & 1 & 603 & 66.22 & 6.51 \\
\hline
\end{tabular}

${ }^{a}$ The information listed in table was obtained from Phytozome 10.1

${ }^{b}$ Names of ARF genes in Carica papaya were based on the nomenclature used in the Arabidopsis model species

'The location of different CpARF genes on each contig or supercontig 

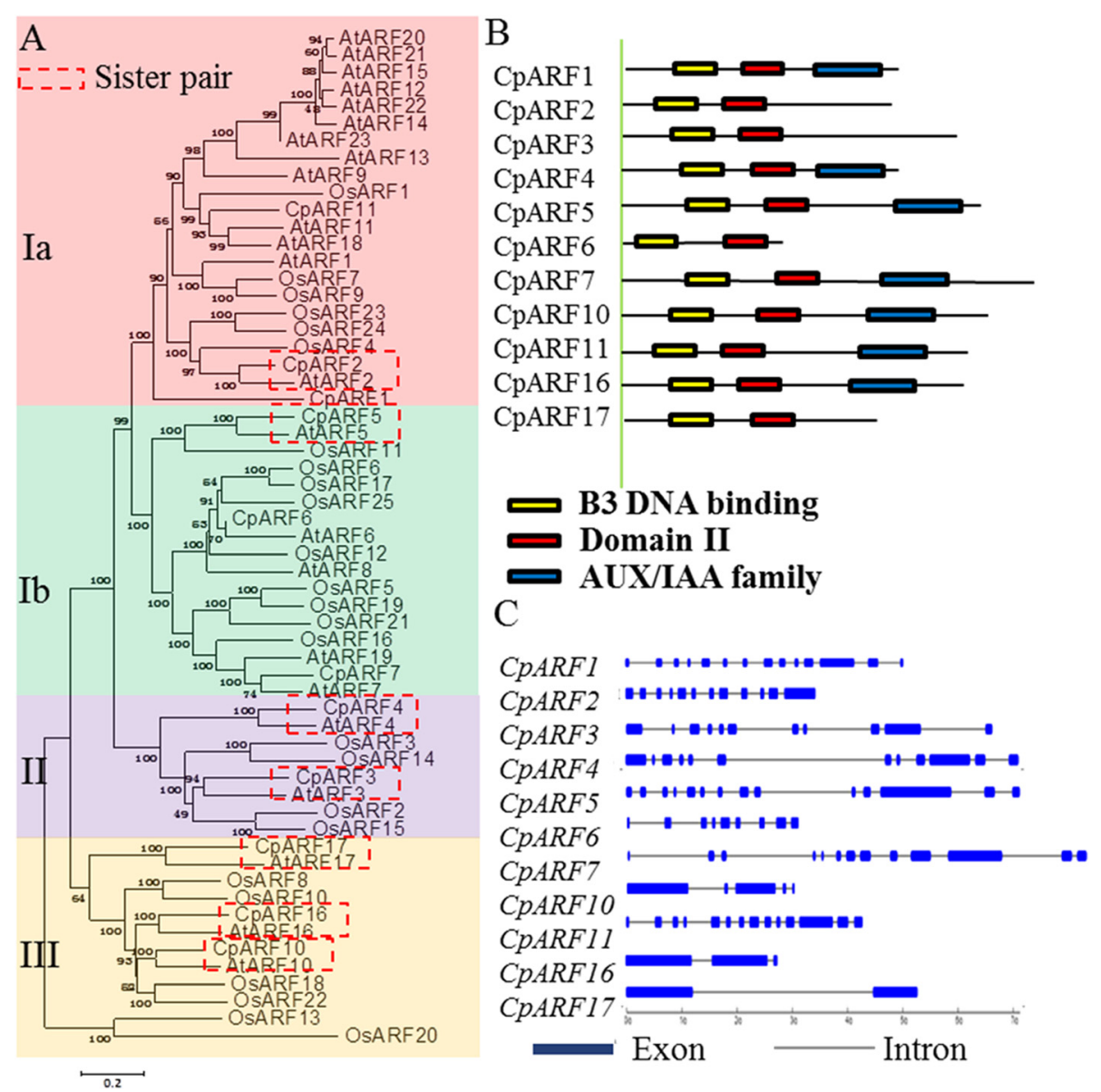

Fig. 1 Analysis of protein domains, gene structures, and phylogenesis. a Phylogeny of auxin response factor (ARF) proteins between different species. Eleven Carica papaya ARFs (CpARFs), 23 Arabidopsis thaliana ARFs (AtARFs), and 25 Oryza sativa ARFs (OsARFs) are classified into three groups: I, II, and III. Group I includes two subgroups: la and Ib. Scale bar 0.1 denotes 0.1 amino-acid substitution per site. $\mathbf{b}$ Schematic organization of CpARFs. The ARF domain, B3 DNA binding domain (DBD), and auxin/indole-3-acetic acid (AUX/IAA) family domain are shown in red, yellow, and blue, respectively.

c Exon-intron structure analysis of CpARF genes. Exons are represented by blue boxes; introns are represented by gray lines

\section{Expression of CpARF genes during flower developmental} stages and fruit set

In our study, we focused on the expression pattern of $\mathrm{CpARF}$ genes in flowers during eight different developmental stages. Except for CPARF17 that showed the lowest expression level in all flowering stages, the remaining $C p A R F$ genes exhibited dynamic expression patterns. CpARF1, CpARF2, CpARF4, CpARF5, and CpARF10 showed the peak expression in flower developmental stage one and decreased during the following developmental stages, while CPARF6 increased during the developmental process and reached the peak at stage seven. In addition, the expression pattern of CpARF genes that belonged to the same phylogenetic branch also varied significantly. The expression of CPARF3 did not change significantly during the developmental process, while the expression of its sister pair gene, CpARF4, showed a clear decrease (Fig. 4 and Additional file 5: Table S4).
Tissue-specific expression analysis showed that some CPARF genes were highly expressed in the reproductive organs (Fig. 3). These results prompted us to investigate the expression of CPARF genes during various fruit ripening stages. The data indicated that the expression of most CPARF genes underwent a significant change associated with fruit ripening. The expression of CPARF1 showed a significant increase during the fruit ripening stages; while the expression of CPARF7 and CPARF11 decreased from stage one to stage six (Fig. 5 and Additional file 6: Table S5).

Auxin regulation of CPARF genes in the flower and fruit The qRT-PCR data showed that most of CpARF genes were responsive to IAA and TIBA treatment.

In flowers, the expression of $C p A R F 1$ was significantly down regulated by IAA treatment and up regulated by TIBA treatment. $C p A R F 2$ and $C p A R F 3$ expression levels 


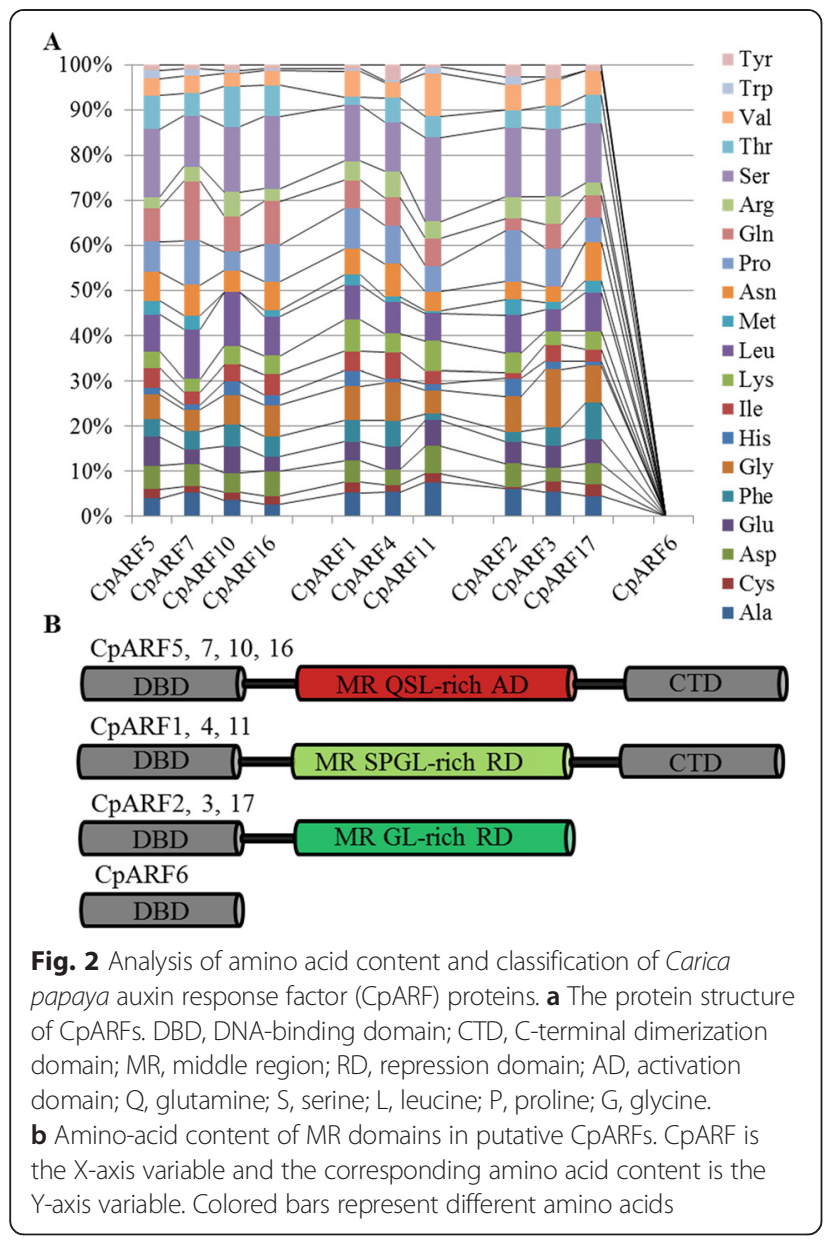

were significantly increased by IAA treatment and remained stable after TIBA treatment. CPARF6 showed no response to IAA treatment and was largely induced by TIBA treatment. CPARF10 also showed no response to IAA treatment and was significantly induced by TIBA treatment. CPARF5 showed opposite expression patterns between IAA treatment and TIBA treatment. The expression of CPARF5 was up regulated by IAA treatment and down regulated by TIBA treatment (Additional file 7: Figure S2).

In fruits, many CpARF genes, such as CpARF1, CpARF4, CPARF5, CPARF7, CPARF11, and CPARF16, were significantly induced by TIBA treatment. However, many CpARF genes, including CPARF2, CpARF4, CPARF6, CPARF10, CPARF16, and CPARF17, were inhibited by IAA treatment (Additional file 8: Figure S3).

\section{Expression of CPARF genes involved in male- hermaphrodite differentiation}

To understand the regulatory mechanisms of auxin signaling involved in sex determination, we analyzed the expression abundance of CPARF genes in the three different sex types. Most CpARF genes showed higher expression abundance in male and hermaphrodite flowers than in female flowers. For example, CPARF3, CPARF6, CPARF11, CPARF16, and CPARF17 showed the highest expression abundance $(>50 \%)$ in male flowers. However, CpARF10 showed the highest expression abundance in hermaphrodite flowers, while it was almost undetectable in male flowers (Fig. 6 and Additional file 9: Table S6).

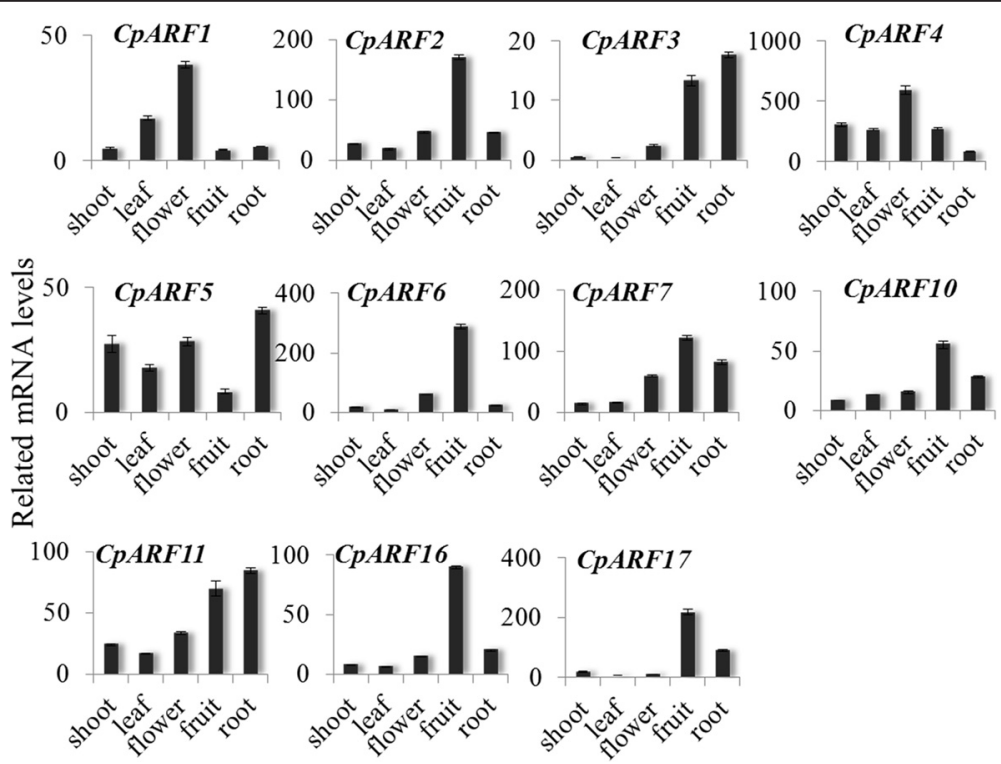

Fig. 3 Tissue-specific expression patterns of Carica papaya auxin response factor (CpARF) genes. Expression pattern of CpARF genes in leaves, shoots, roots, flowers, and fruits of 2-year-old papaya plants. CPACTIN value is 1000 . Means are from five independent repeats; error bars show standard deviations 


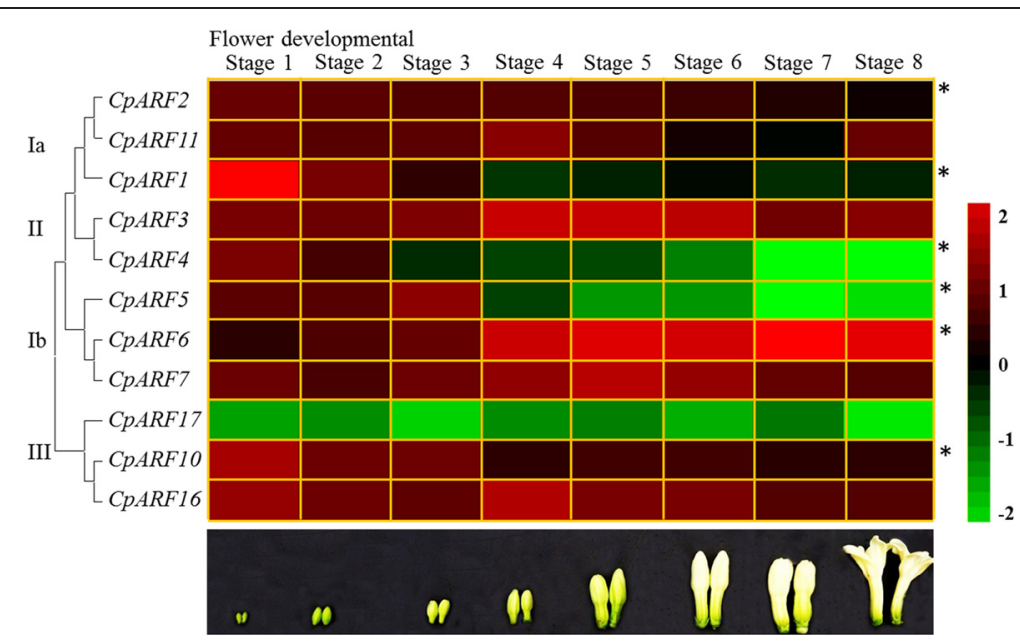

Fig. 4 Heatmap of Carica papaya auxin response factor (CPARF) gene expression during different flower developmental stages. Changes in the expression levels during different flower developmental stages that schematically depicted above the displayed quantitative real time (qRT) data are relative to RNA accumulation levels. Levels of down expression (green) or up expression (red) are shown on a log2 scale from the highest to the lowest expression of each CPARF gene. Significant $(P<0.05)$ differences are indicated by an asterisk

Analysis of AuxREs in the promoter of reproduction-related genes

After searching the papaya genome database, we selected seven floral meristem determinacy related homologous genes (Class A-E) [43, 44], nine CpKNOX genes (CpKNOX1-CpKNOX9), four flower development-related homologous genes (CpFT1-3 and CpLFY1) [45, 46], four ethylene-signaling-related homologous genes $(C p E T R 1 / 2$ and $C p C T R 1 / 2)[47,48]$, and three ethylene-synthesisrelated homologous genes ( $C p A C S 1 / 2$ and $C p A C O 1)$ [49] for this analysis. Among the 27 selected gene promoters,
16 promoters contained one or more AuxREs (AUX1 or 2) (Fig. 7 and Additional file 10: Table S7). Therefore, it was suggested that some reproduction-related genes could be strongly regulated by auxin treatment. All the promoter sequences of reproduction-related genes were listed in Additional file 11: Table S8.

\section{Endogenous IAA measurement}

To reveal the involvement of auxin in the development of flowers and fruits in papaya, endogenous IAA contents were measured. The data showed that

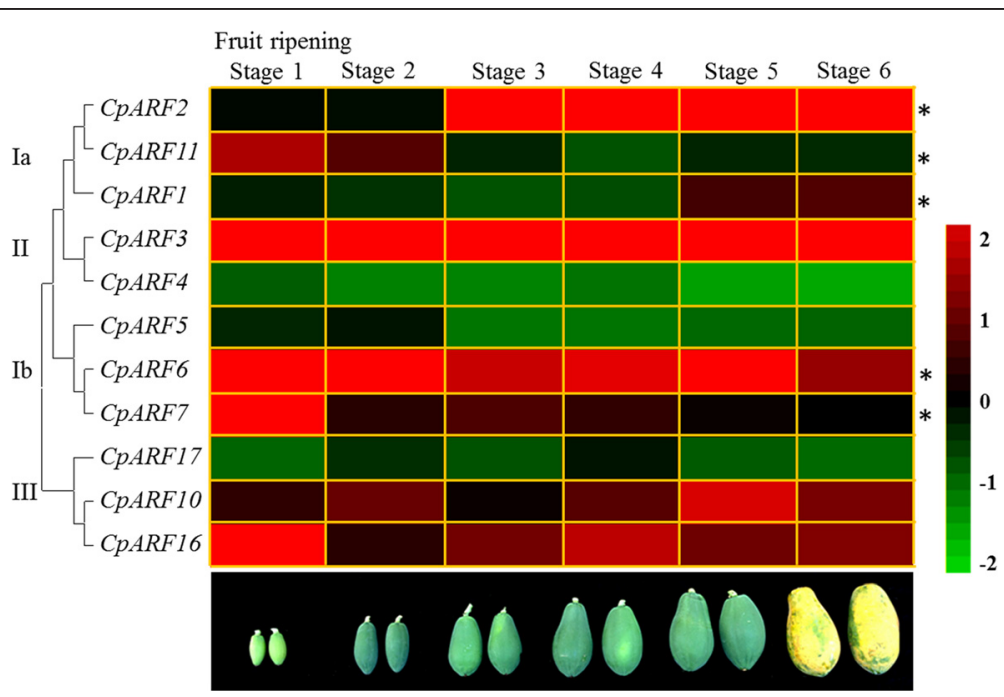

Fig. 5 Heatmap of Carica papaya auxin response factor (CPARF) gene expression during different fruit developmental stages. Changes in the expression levels during different fruit developmental stages that schematically depicted above the displayed quantitative real time (qRT) data are relative to RNA accumulation levels. Levels of down expression (green) or up expression (red) are shown on a log2 scale from the highest to the lowest expression of each CPARF gene. Significant $(P<0.05)$ differences are indicated by an asterisk 


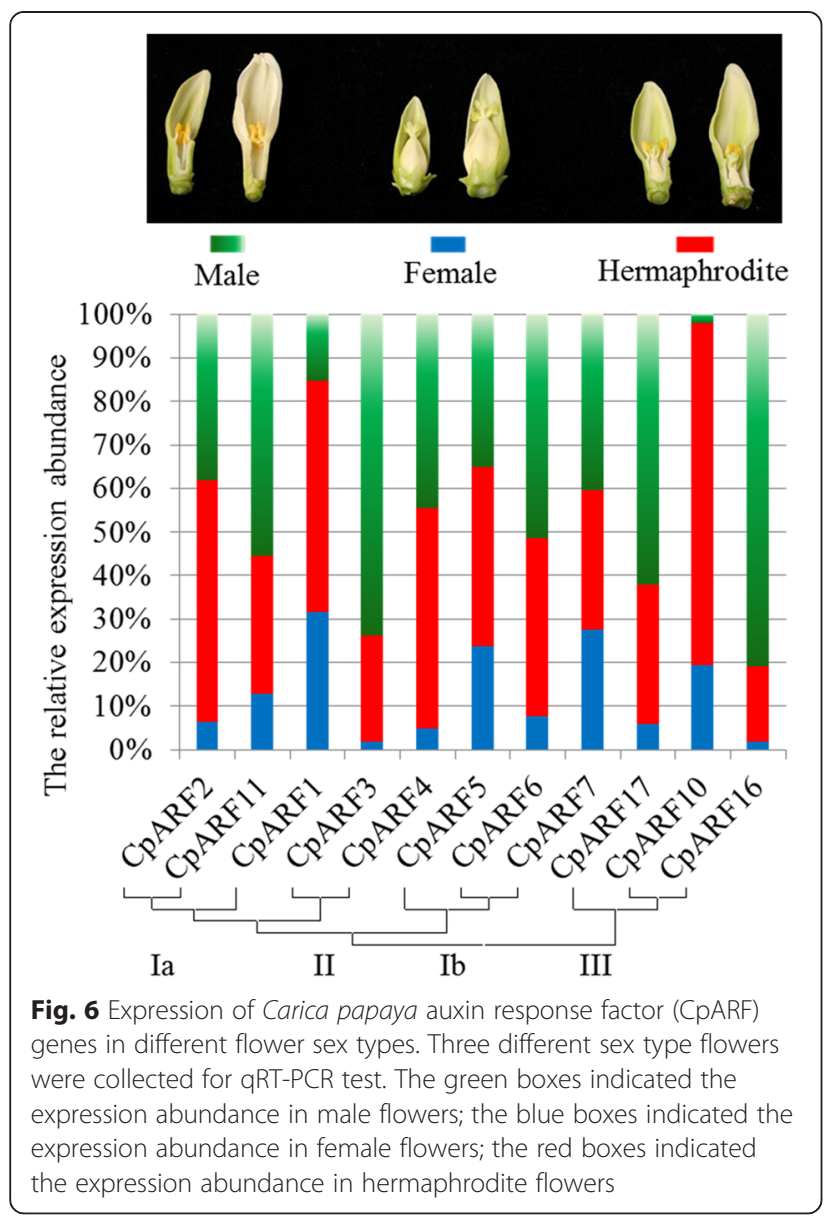

the endogenous IAA contents were much lower in the flowers under later stages than in the flowers under early stages. In the fruits, the endogenous IAA contents keep on a high level from stage one to stage four, and then significantly declined in the stages five and six. Furthermore, three different sex type flowers were collected for endogenous IAA measurements. The highest IAA contents were detected in the male flowers. The IAA contents in the female and hermaphrodite flowers were lower than that in the male flowers (Additional file 12: Figure S4).

\section{Discussion}

Auxin is a key signaling molecule for most organogenesis and patterning processes occurring during plant development [50]. The auxin transduction pathway is mainly comprised of two transcriptional regulator families: ARFs and Aux/IAAs [37, 51]. ARFs directly bind to down-stream target genes and regulate their expression during development [52]. ARFs are also involved in the reproduction of various plant species [3, 53]. Characterization and analysis of CpARFs allowed us to reveal the mechanisms behind auxin involvement in fruit and flower development of papaya [54].

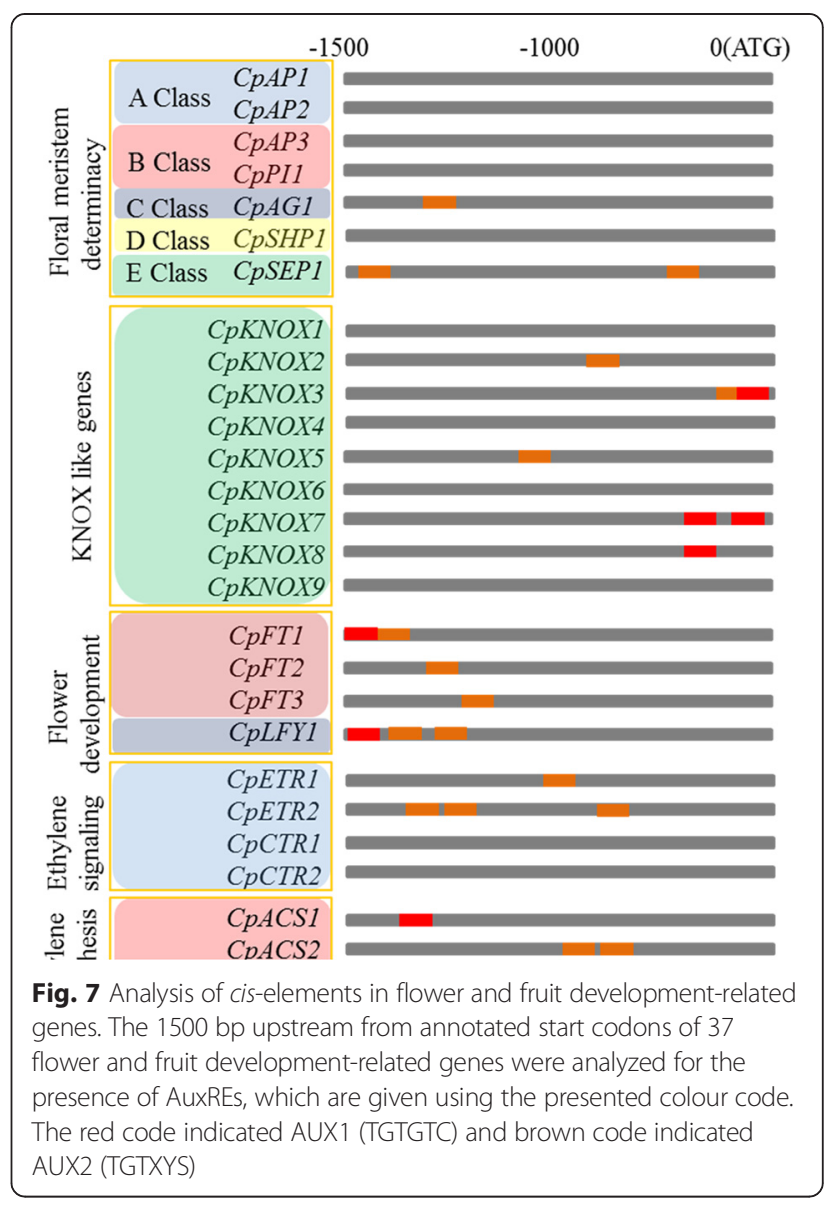

In this study, the reference genome sequence of papaya, which is relatively small in size (372 Mbp) [55], was used to identify the complete $C p A R F$ family. The number of CpARF genes was less than that in Arabidopsis (23 ARFs) [37]. Protein domain analysis provided us useful information on the biological function of ARFs. A typical ARF contains a DBD, an MR, and a CTD [37]. Aux/IAAs bind to CTDs of ARFs and form heterodimers. The presence of a large number of CPARFs without CTD suggested that some auxin-responsive genes in papaya can be regulated in an auxin independent manner [51]. The percentage of CTD-truncated CpARFs $(36.4 \%)$ was higher than that in other plant species, such as soybean (15.68\%), Arabidopsis (17.39\%), Brassica rapa (22.58\%), rice (24\%), and tomato (28.57\%) $[10,56]$. Based on the amino acid composition of MR domains, CpARFs were classified into two groups: transcriptional activators and repressors [8]. The average activator/repressor ratio of CpARFs was 0.57 (Fig. 2), similar to Arabidopsis (0.59) and rice (0.56), and almost double compared to that in tomato (0.27) [5]. Only one ARF in papaya, CpARF6, contained only a DBD. These data provided insight into the potential functions of CpARF genes in plant developmental regulation. 
We also built a phylogenetic tree to analyze the relationship of ARF families between papaya, Arabidopsis, and rice. The results showed that seven sister gene pairs with high bootstrap values ( $\geq 99 \%$ ) were identified between papaya and Arabidopsis, suggesting that ARFs in papaya were highly homologous to those in Arabidopsis (Fig. 1a). Many AtARFs have been already reported in previous reports [53, 57-59]; therefore, comparative studies may reveal useful information on the respective biological functions in papaya.

In Arabidopsis, transcription factors ARF6 and ARF8 regulate a complex process by promoting expansion, stamen filament elongation, anther dehiscence, and gynoecium maturation $[18,50]$. The expression of CPARF6, a homologous gene of AtARF6 and AtARF8, was increased more than six folds from flower developmental stage 1 to stage 8 (Fig. 4), indicating a putative function of this gene in flower development and maturation. The double mutant arf6/arf8 in Arabidopsis delays the elongation of floral organs and subsequently delays the opening of flower buds and petal growth $[18,60]$. Most defects in arf6/arfs are attributed to the abnormal expression of class one KNOXs [61]. The promoters of some KNOX genes in papaya, such as $C p K N O X 2,3,5$, 7 , and 8 , contain several AuxRE elements, suggesting that these genes may be negatively regulated by CPARF6 in the developing floral organs (Fig. 7).

AtARF4 was also reported to be involved in flower patterning [62]. CPARF4 (homologous gene of AtARF4) showed high expression levels in the flowers (Fig. 3). However, the expression of CPARF4 gradually declined from flower developmental stage one to stage eight, suggesting that it might play a different role compared to CPARF6 during flower development, especially at the initial stage. The Arabidopsis mutant arf2 has a delayed flowering and ripening, while a double mutant arf1/arf2 has an enhanced arf2 phenotype, indicating that AtARF1 acts in a partially redundant manner with AtARF2 [53]. In papaya, CPARF1 (homologous gene of AtARF1) was highly expressed in flowers, while $C P A R F 2$ (homologous gene of AtARF2) showed a fruit-specific expression. Furthermore, the expression level of CPARF1 was much higher in female flowers than in male flowers, and CPARF2 showed an opposite expression pattern to CPARF1. The expression level of CPARF2 was eight-fold higher in male flowers than in female flowers (Fig. 6). Additionally, the expression of CPARF1 and CPARF2 also declined during flower development (Fig. 4). The preferred expression in early stages suggested that CPARF1 and CPARF2 participated in flower bud formation, which is a key step for flower development. TIR1/AFB-mediated auxin-responsive gene expression is controlled by the interaction between Aux/IAA repressors and ARF transcription factors [63]. CpARF1,
CPARF2, and CpARF4-related auxin expression regulation was decreased, while CPARF6-mediated auxin expression regulation was activated in the mature flowers.

Fruit development is a complex interplay of cell division, differentiation, and expansion that occurs in a temporally and spatially coordinated manner in the reproductive organs [64]. Auxin triggers and/or promotes the unpollinated, quiescent ovary to undergo cell division and elongation, and hence it is considered to play a major role in fruit set and development $[65,66]$. In tomato, SIARFs are involved in the regulation of various aspects of fruit development [67]. SIARF7 acts as a negative regulator of fruit set after pollination and fertilization, and moderates auxin response during fruit growth [68]. Another tomato gene, SlARF4, an auxin response factor involved in the control of sugar metabolism during fruit development, expresses in pericarp tissues of immature fruit [26]. In papaya, several $C p A R F$ genes, including CPARF2, CpARF6, CpARF7, CPARF10, $C P A R F 16$, and $C P A R F 17$, displayed fruit-specific expression patterns, suggesting their importance in improving fruit-related agronomic traits in papaya [29]. Goetz et al. suggested that AtARF8 restricts auxin signal transduction in ovules and pistil until the initiation of fruit development [12]. However, no homologous gene of AtARF8 was identified in papaya.

It is well studied that reproductive organs of plants reacted differently to different plant hormones. Many previous researches have presumed that auxin might play important roles in flower differentiation in papaya, and delay fruit ripening in other plant species $[34,36]$. However, there is still no decisive evidence revealing that endogenous IAA plays roles in the flower and fruit development in papaya. The endogenous IAA contents showed a decline during both the flower and fruit development, suggesting that a high level of endogenous IAA might contribute to the initiation of reproductive organs in papaya.

Ethylene-auxin crosstalk regulates a variety of developmental and growth processes in plants, including fruit development and ripening [69-73]. Auxin plays a key role in progressing of fruit development towards the transition phase that leads to the initiation of autocatalytic ethylene production in an auxin- and ethylene-dependent manner [73-75]. In Arabidopsis, AtARF7 and AtARF19 are involved in ethylene response, indicating an interaction between auxin and ethylene [58]. SlARF7, a homolog of AtARF7 in tomato, was also found to be involved in auxin signaling transduction during tomato fruit set and development [76]. In our study, the expression of CPARF7 (homologous gene of AtARF7) was significantly inhibited during fruit ripening (Fig. 5). High expression levels of CPARF6 and 
CPARF7 in mature flowers and early fruit developmental stages indicated that these two genes might be involved in fruit set and early cell division stage of the fruit. To get the putative targets for CpARFs during fruit ripening, we analyzed the promoter regions of several ethylene-signaling-and ethylene-synthesis-related genes in papaya [43-49]. The results showed that many AuxREs were contained in the promoters of two selected ethylene-signaling-related genes (CPETR1 and $C p E T R 2)$ and three ethylene-synthesis-related genes (CpACS1, CpACS2 and CpACO1) (Fig. 7). In papaya, ARFs may be also involved in fruit ripening by regulating ethylene-signaling-related and ethylene-synthesisrelated genes.

\section{Conclusions}

In conclusion, our study provided comprehensive information on $A R F$ family in papaya, including gene structures, chromosome locations, phylogenetic relationships, and expression patterns. The involvement of CpARF gene expressions in flower and fruit development allowed us to understand the role of ARFmediated auxin signaling in the maturation of reproductive organs in papaya.

\section{Methods}

\section{Plant materials and growth conditions}

Two-year-old C. papaya cv. 'Sunrise' trees were planted in a $3 \mathrm{~m} \times 3-\mathrm{m}$ plot with drip irrigation at the Lingnan Normal University field experimental station in Zhanjiang City, Guangdong Province, China. Agronomic practices and fertilizer applications were applied as needed. Our experimental station has a gentle tropical oceanic monsoon climate with an average daily temperature of $22.8^{\circ} \mathrm{C}$, minimum temperature of $15.7{ }^{\circ} \mathrm{C}$, and maximum temperature of $28.8^{\circ} \mathrm{C}$. The total yearly rainfall ranges between 1100 and $1800 \mathrm{~mm}$ [77]. The environmental conditions were strictly recorded during the sampling period. No extreme events and bad weather occurred in our experiment period.

\section{Genome-wide identification of CpARF genes}

Arabidopsis ARFs (AtARFs) were used to blast against the C. papaya genome database on Phytozome 10.1 using TBLASTN (http://phytozome.jgi.doe.gov). Information on AtARFs used in this study is presented in Additional file 13: Table S9. Furthermore, the hidden Markov model (HMM) profiles of the ARF family [Pfam 02309: AUX/IAA family; Pfam 06507: ARF (AUX_RESP); Pfam 02362: DBD] were employed to identify $A R F \mathrm{~s}$ from the $C$. papaya genome. All the obtained sequences were sorted as unique sequences for further protein domain search using InterProScan (http://www.ebi.ac.uk/Tools/pfa/iprscan/).

\section{Sequence analysis and phylogenetic tree}

Multiple sequence alignment of CpARFs was performed using ClustalW (http://www.ebi.ac.uk/Tools/msa/ clustalw2/) with the default parameters and adjusted manually. Four classical domains were identified in most CpARFs based on alignment results. DNA and cDNA sequences corresponding to each predicted gene were obtained from the $C$. papaya genome. Arabidopsis and rice $A R F \mathrm{~s}$ (OsARFs) were used for the construction of a phylogenetic tree. Information on $A t A R F \mathrm{~s}$ and OsARFs is presented in Additional file 13: Table S9. Gene structure was analyzed using Gene Structure Display Server (http://gsds.cbi.pku.edu.cn/ index.php), and the phylogenetic tree was constructed with 11 aligned CPARF sequences, 23 AtARF sequences, and 25 OsARF sequences using MEGA5.1 (http://www.megasoftware.net/) employing the neighborjoining (NJ) method. Bootstrap values were calculated using 1000 iterations. The constructed phylogenetic tree was visualized using TreeView1.6 (http://www.brc.dcs. gla.ac.uk/services/).

\section{Prediction of amino-acid content and protein classification}

Amino-acid content of the MR domain in CpARFs was calculated using MEGA 5.1, and the histogram was constructed using Excel 2010. The classification of CpARFs was based on the respective amino acid content [Domains with CTD: Glutamine/serine/leucine (QSL)rich MR; Repressor with a carboxyl terminal domain (CTD); Serine/proline/glycine/leucine (SPGL)-rich MR; Repressor without CTD: Glycine-rich MR].

\section{RNA isolation and quantitative real time polymerase chain reaction (qRT-PCR)}

Total RNA from different tissues, such as shoots, leaves, flowers, fruits, and roots, was extracted using Plant RNeasy Mini kit (Qiagen, Hilden, Germany) according to the manufacturer's instructions. The criterion of flowers and fruits under different developmental stages was described as follows. In total, flowers of eight different developmental stages were collected in this experiment, including five stages of flower buds based on their diameters $(1 \mathrm{~mm}$, stage $1 ; 3 \mathrm{~mm}$, stage 2 ; $5 \mathrm{~mm}$, stage $3 ; 7 \mathrm{~mm}$, stage 4 and $9-10 \mathrm{~mm}$, stage 5 ), young flower with closed petals (stage 6), mature flower with partially opened petals (stage 7) and mature flower with opened petals (stage 8). In addition, papaya fruit samples of different developmental stages were harvested at 20,40, 60, 80, 100 and 120 days after anthesis, respectively. For all the fruit samples, fruit core was excluded, and the flesh with peel were chopped up, frozen in liquid nitrogen and stored at $-80{ }^{\circ} \mathrm{C}$ for further test. Flowers used in tissue-specific expression experiment 
were a mixture of male, female, and hermaphrodite types. To avoid the affects of environmental factors, the fruit and flower samples were collected from fifteen of uniform, well growth and disease free trees that distributed in different places in our field. Then, the samples were mixed and divided into several independent groups for further analysis.

DNase I was used to remove any genomic DNA contamination from total RNA. CpActin (evm.model.supercontig_18.238) was used as an internal standard to calculate the relative fold differences based on the comparative cycle threshold $\left(2^{-\Delta \Delta C t}\right)$ values. Briefly, $1 \mu \mathrm{l}$ of $1 / 20$ dilution of cDNA was mixed with $5 \mu \mathrm{l}$ of $2 \times$ SYBGreen and $100 \mathrm{nM}$ of each primer (forward and reverse), and then water was added to a final volume of $10 \mu$ l. PCR conditions were as follows: $95{ }^{\circ} \mathrm{C}$ for $10 \mathrm{~min}, 40$ cycles at $95{ }^{\circ} \mathrm{C}$ for $15 \mathrm{~s}$, and $60{ }^{\circ} \mathrm{C}$ for $60 \mathrm{~s}$. All the primer sequences are listed in Additional file 14: Table S10. To visualize qRT-PCR data, heat map was constructed by ClustalW and Treeview using the average $C t$ value. In the heat map, red color represented up regulation, black color represented unchanged expression, and green color represented down regulation. In this experiment, a specific fold change value $(2 \times)$ was used to identify any significant differences between different treatments. Expression analysis was carried out using five biological repeats, and the values shown in figures represent the average values of the five repeats.

\section{IAA treatment and cis-elements analysis}

Flower and fruit samples were soaked in liquid Murashige and Skoog (MS) medium with or without (mock treatment) $10 \mu \mathrm{M}$ IAA or $10 \mathrm{iM} \mathrm{2,} \mathrm{3,} \mathrm{5-}$ triodobenzoic acid (TIBA) for $1 \mathrm{~h}$. Samples from each treatment were collected, and total RNA was isolated as previously described. Experiments were repeated five times with similar results. The promoters (1500 bp) of reproduction-related genes were obtained from Phytozome 10.1. AUX1 (TGTCTC core sequence) and a less stringent variant called AUX2 (TGTVYS) were used to manually scan promoter regions.

\section{IAA content measurement}

The fruit and flower samples were collected and washed five times in deionized water to clean the surface of the tissues. The plant tissues were blotted dry with a paper towel and weighed using an electronic balance. After the addition of $500 \mathrm{pg}$ of the ${ }^{13} \mathrm{C} 6$-IAA internal standard, five independent biological replicates of each $50 \mathrm{mg}$ sample were purified using ProElu C18 (http://www.dikma.com.cn). IAA contents were determined by a FOCUS GC-DSQII (Thermo Fisher Scientific Inc., Austin, TX, USA).

\section{Availability of supporting data}

All the supporting data are included as Additional files.

\section{Additional files}

Additional file 1: Table S1. The nucleic acid sequences of CPARF family genes. (DOCX $23 \mathrm{~kb}$ )

Additional file 2: Figure S1. Multiple alignment profile of CpARF proteins obtained with ClustalW program. Multiple alignments of the DBD, MR and CTD domains of the CpARF proteins also were showed by different color lines. Colorized shading indicates identical and conversed amino acid residues, respectively. Two NLSs were marked by black asterisks. (TIF $3259 \mathrm{~kb}$ )

Additional file 3: Table S2. Domain positions in 11 CpARF proteins (DOCX $16 \mathrm{~kb}$ )

Additional file 4: Table S3. Data of amino acid content in MR domain of CpARFs. (DOCX $19 \mathrm{~kb}$ )

Additional file 5: Table S4. The saw data of the CPARF family during the different flower developmental stages. (XLSX $10 \mathrm{~kb}$ )

Additional file 6: Table S5. The saw data of the CPARF family during the different fruit developmental stages. (XLSX $10 \mathrm{~kb}$ )

Additional file 7: Figure S2. The expression level of CPARF genes under IAA and TIBA treatments in flowers. The histogram shows the relative expression level of CDARF genes under IAA and TIBA treatments during different time points compared to the mock expression level. Significant $(P<0.05)$ differences in control and treatments are indicated by an asterisk. (TIF $424 \mathrm{~kb}$ )

Additional file 8: Figure S3. The expression level of CPARF genes under IAA and TIBA treatments fruits. The histogram shows the relative expression level of CDARF genes under IAA and TIBA treatments during different time points compared to the mock expression level. Significant $(P<0.05)$ differences in control and treatments are indicated by an asterisk. (TIF $407 \mathrm{~kb}$ )

Additional file 9: Table S6. The saw data of the CpARF family during the different fruit developmental stages. (XLSX $10 \mathrm{~kb}$ )

Additional file 10: Table S7. Promoter analysis (locations from ATG) of the genes involved in flower development and fruit ripening. (DOCX $16 \mathrm{~kb}$ )

Additional file 11: Table S8. The promoter sequences of reproductionrelated genes. (DOCX $28 \mathrm{~kb}$ )

Additional file 12: Figure S4. The endogenous IAA contents measurment. (a) The endogenous IAA contents in the flowers under different developmental stages. (b) The endogenous IAA contents in the fruits under different developmental stages. (c) The endogenous IAA contents in the flowers of different sex types. Significant $(P<0.05)$ differences in IAA contents are indicated by an asterisk. (TIF $516 \mathrm{~kb}$ )

Additional file 13: Table S9. The information of ARF family gene in Arabidopsis and rice. (DOCX $18 \mathrm{~kb}$ )

Additional file 14: Table S10. Primer sequences for qRT-PCR of CPARF family genes. (DOCX $17 \mathrm{~kb}$ )

\section{Abbreviations}

ARF: Auxin response factor; Aux/IAA: Auxin/indole-3-acetic acid; AuxRE: Auxin response element; CTD: C-terminal dimerization domain; DBD: DNA binding domain; GH3: Gretchen Hagen3; HMM: Hidden Markov model; kDa: k Dalton; SAUR: Small auxin up RNA; MR: Middle region; MS: Murashige and Skoog; ORF: Open reading frame; IAA: Indole-3-acetic acid; TIBA: 2, 3, 5-triodobenzoic acid; GRT-PCR: Quantitative real-time polymerase chain reaction; TBLAST: Basic local alignment search tool.

\section{Competing interests}

The authors declare that they have no competing interests. 


\section{Authors' contributions}

$\mathrm{KDL}, \mathrm{CJS}$ and XLZ conceived and designed the study; KDL, CCY, and HLL performed laboratory experiments; KDL and WHL performed the data analysis; CJS and YJY assisted in the data analysis; KDL wrote the manuscript with assistance from CJS; All authors read and approved the final manuscript.

\section{Acknowledgements}

This work was supported by the National Natural Science Foundation of China (grant no. 31201586 and 31401935); Science and Technology Program of Guangdong, China (grant no. 2014A020208138 and 2015A020208018); the (Key) Project of Department of Education of Guangdong Province (grant no. 2013KJCX0124). The authors thank two anonymous reviewers whose comments greatly improved the final manuscript. The authors also thank Prof. Langtao Xiao from Hunan Agricultural University, Prof. Jinxiang Wang and Jiang Tian from South China Agricultural University, and Prof. Rongchen Wang from Huazhong Agricultural University for their valuable comments.

\section{Author details}

${ }^{1}$ College of Bioscience and Technology, Hunan Agricultural University, Changsha, Hunan 410128, China. ${ }^{2}$ College of Food Science and Biotechnology, Zhejiang Gongshang University, Hangzhou 310035, China. ${ }^{3}$ College of Life and Environmental Sciences, Hangzhou Normal University, Hangzhou 310036, China. ${ }^{4}$ Life Science and Technology School, Lingnan Normal University, Zhanjiang, Guangdong 524048, China.

\section{Received: 5 June 2015 Accepted: 30 October 2015} Published online: 05 November 2015

\section{References}

1. Woodward AW, Bartel B. Auxin: regulation, action, and interaction. Ann Bot. 2005;95(5):707-35.

2. Ljung K. Auxin metabolism and homeostasis during plant development. Development. 2013;140(5):943-50.

3. Kumar R, Tyagi AK, Sharma AK. Genome-wide analysis of auxin response factor (ARF) gene family from tomato and analysis of their role in flower and fruit development. Mol Gen Genet MGG. 2011;285(3):245-60.

4. Shen C, Yue R, Yang Y, Zhang L, Sun T, Xu L, et al. Genome-wide identification and expression profiling analysis of the Aux/IAA gene family in Medicago truncatula during the early phase of Sinorhizobium meliloti infection. PLoS One. 2014;9(9):e107495.

5. Zouine M, Fu Y, Chateigner-Boutin AL, Mila I, Frasse P, Wang H, et al. Characterization of the tomato ARF gene family uncovers a multi-levels post-transcriptional regulation including alternative splicing. PLOS One. 2014;9(1):e84203.

6. Guilfoyle TJ, Ulmasov T, Hagen G. The ARF family of transcription factors and their role in plant hormone-responsive transcription. Cell Mol Life Sci. 1998;54(7):619-27.

7. Shen C, Yue R, Sun T, Zhang L, Xu L, Tie S, et al. Genome-wide identification and expression analysis of auxin response factor gene family in Medicago truncatula. Frontiers Plant Sci. 2015;6:73.

8. Tiwari SB, Hagen G, Guilfoyle T. The roles of auxin response factor domains in auxin-responsive transcription. Plant Cell. 2003;15(2):533-43.

9. Hagen G, Guilfoyle T. Auxin-responsive gene expression: genes, promoters and regulatory factors. Plant Mol Biol. 2002;49(3-4):373-85.

10. Shen C, Wang S, Bai Y, Wu Y, Zhang S, Chen M, et al. Functional analysis of the structural domain of ARF proteins in rice (Oryza sativa L.). J Exp Bot. 2010:61(14):3971-81.

11. Ulmasov T, Hagen G, Guilfoyle TJ. Activation and repression of transcription by auxin-response factors. Proc Natl Acad Sci U S A. 1999;96(10):5844-9.

12. Goetz M, Vivian-Smith A, Johnson SD, Koltunow AM. AUXIN RESPONSE FACTOR8 is a negative regulator of fruit initiation in Arabidopsis. Plant Cell. 2006;18(8):1873-86.

13. Sessions A, Nemhauser JL, McColl A, Roe JL, Feldmann KA, Zambryski PC. ETTIN patterns the Arabidopsis floral meristem and reproductive organs. Development. 1997;124(22):4481-91.

14. Nishimura T, Wada T, Yamamoto KT, Okada K. The Arabidopsis STV1 protein, responsible for translation reinitiation, is required for auxin-mediated gynoecium patterning. Plant Cell. 2005;17(11):2940-53.

15. Hardtke CS, Berleth T. The Arabidopsis gene MONOPTEROS encodes a transcription factor mediating embryo axis formation and vascular development. EMBO J. 1998;17(5):1405-11.
16. Harper RM, Stowe-Evans EL, Luesse DR, Muto H, Tatematsu K, Watahiki MK, et al. The NPH4 locus encodes the auxin response factor ARF7, a conditional regulator of differential growth in aerial Arabidopsis tissue. Plant Cell. 2000;12(5):757-70.

17. Tian CE, Muto H, Higuchi K, Matamura T, Tatematsu K, Koshiba T, et al. Disruption and overexpression of auxin response factor 8 gene of Arabidopsis affect hypocotyl elongation and root growth habit, indicating its possible involvement in auxin homeostasis in light condition. Plan0074 J: for cell and Mol Biol. 2004;40(3):333-43.

18. Nagpal P, Ellis CM, Weber H, Ploense SE, Barkawi LS, Guilfoyle TJ, et al. Auxin response factors ARF6 and ARF8 promote jasmonic acid production and flower maturation. Development. 2005;132(18):4107-18.

19. Narise T, Kobayashi K, Baba S, Shimojima M, Masuda S, Fukaki H, et al. Involvement of auxin signaling mediated by IAA14 and ARF7/19 in membrane lipid remodeling during phosphate starvation. Plant Mol Biol. 2010;72(4-5):533-44.

20. Attia KA, Abdelkhalik AF, Ammar MH, Wei C, Yang J, Lightfoot DA, et al. Antisense phenotypes reveal a functional expression of OsARF1, an auxin response factor, in transgenic rice. Curr Issues Mol Biol. 2009;11 Suppl 1:i29-34.

21. Shen C, Wang S, Zhang S, Xu Y, Qian Q, Qi Y, et al. OsARF16, a transcription factor, is required for auxin and phosphate starvation response in rice (Oryza sativa L.). Plant Cell Environ. 2013;36(3):607-20.

22. Shen $C$, Yue R, Sun T, Zhang L, Yang Y, Wang H. OsARF16, a transcription factor regulating auxin redistribution, is required for iron deficiency response in rice (Oryza sativa L.). Plant sci: Int J Exp plant bio. 2015;231:148-58.

23. Shen C, Yue R, Yang Y, Zhang L, Sun T, Tie S, et al. OsARF16 is involved in cytokinin-mediated inhibition of phosphate transport and phosphate signaling in rice (Oryza sativa L.). PLoS One. 2014;9(11):e112906.

24. Zhang S, Wang S, Xu Y, Yu C, Shen C, Qian Q, et al. The auxin response factor, OsARF19, controls rice leaf angles through positively regulating OsGH3-5 and OsBR/1. Plant Cell Environ. 2014;38:638-54.

25. Sagar M, Chervin C, Roustant JP, Bouzayen M, Zouine M: Under-expression of the Auxin Response Factor SI-ARF4 improves postharvest behavior of tomato fruits. Plant signaling \& behavior 2013, 8(10):doi: 10 4161/psb 25647

26. Sagar M, Chervin C, Mila I, Hao Y, Roustan JP, Benichou M, et al. SIARF4, an auxin response factor involved in the control of sugar metabolism during tomato fruit development. Plant Physiol. 2013;161(3):1362-74.

27. Kumar R, Agarwal P, Tyagi AK, Sharma AK. Genome-wide investigation and expression analysis suggest diverse roles of auxin-responsive GH3 genes during development and response to different stimuli in tomato (Solanum lycopersicum). Mol Gen Genet MGG. 2012;287(3):221-35.

28. Urasaki N, Tarora K, Shudo A, Ueno H, Tamaki M, Miyagi N, et al. Digital transcriptome analysis of putative sex-determination genes in papaya (Carica papaya). PLoS One. 2012;7(7):e40904.

29. Ueno H, Urasaki N, Natsume S, Yoshida K, Tarora K, Shudo A, et al. Genome sequence comparison reveals a candidate gene involved in male-hermaphrodite differentiation in papaya (Carica papaya) trees. Mol Gen Genet MGG. 2015;290(2):661-70.

30. Aryal R, Ming R. Sex determination in flowering plants: papaya as a model system. Plant sci: Int J Exp Plant Bio. 2014;217-218:56-62.

31. Dellaporta SL, Calderon-Urrea A. Sex determination in flowering plants Plant Cell. 1993;5(10):1241-51

32. Deputy JC, Ming R, Ma H, Liu Z, Fitch MM, Wang M, et al. Molecular markers for sex determination in papaya (Carica papaya L.). TAG. 2002;106(1):107-11.

33. Tamaki M, Urasaki N, Sunakawa Y, Motomura K, Adaniya S. Seasonal variations in pollen germination ability, reproductive function of pistils, and seeds and fruit yield in papaya (Carica papaya L.) in Okinawa. J Jpn Soc Hortic Sci. 2011;80(2):156-63.

34. Jones B, Frasse P, Olmos E, Zegzouti H, Li ZG, Latche A, et al. Downregulation of $D R 12$, an auxin-response-factor homolog, in the tomato results in a pleiotropic phenotype including dark green and blotchy ripening fruit. Plant J: cell and Mol Biol. 2002;32(4):603-13.

35. PÉRez-Carrillo E, Yahia EM. Effect of postharvest Hot Air and fungicide treatments on the quality of 'Maradol' papaya (CARICA PAPAYA L.). J Food Qual. 2004;27(2):127-39.

36. Chavez-Sanchez I, Carrillo-Lopez A, Vega-Garcia M, Yahia EM. The effect of antifungal hot-water treatments on papaya postharvest quality and activity of pectinmethylesterase and polygalacturonase. J Food Sci Tech Mys. 2013;50(1):101-7. 
37. Guilfoyle TJ, Hagen G. Auxin response factors. Curr Opin Plant Biol. 2007;10(5):453-60.

38. Liu PP, Montgomery TA, Fahlgren N, Kasschau KD, Nonogaki H, Carrington JC. Repression of AUXIN RESPONSE FACTOR10 by microRNA160 is critical for seed germination and post-germination stages. Plant J:cell and Mol Biology. 2007:52(1):133-46.

39. Wang D, Pei K, Fu Y, Sun Z, Li S, Liu H, et al. Genome-wide analysis of the auxin response factors (ARF) gene family in rice (Oryza sativa). Gene. 2007;394(1-2):13-24.

40. Kalluri UC, Difazio SP, Brunner AM, Tuskan GA. Genome-wide analysis of Aux/IAA and ARF gene families in Populus trichocarpa. BMC Plant Biol. 2007;7:59.

41. Wang Y, Deng D, Shi Y, Miao N, Bian Y, Yin Z. Diversification, phylogeny and evolution of auxin response factor (ARF) family: insights gained from analyzing maize ARF genes. Mol Biol Rep. 2012;39(3):2401-15.

42. Li SB, OuYang WZ, Hou XJ, Xie LL, Hu CG, Zhang JZ. Genome-wide identification, isolation and expression analysis of auxin response factor (ARF) gene family in sweet orange (Citrus sinensis). Frontiers in plant sci. 2015;6:119.

43. Jofuku KD, den Boer BG, Van Montagu M, Okamuro JK. Control of Arabidopsis flower and seed development by the homeotic gene APETALA2. Plant Cell. 1994;6(9):1211-25.

44. Bowman JL, Smyth DR, Meyerowitz EM. Genes directing flower development in Arabidopsis. Plant Cell. 1989;1(1):37-52

45. Engelhorn J, Moreau F, Fletcher JC, Carles CC. ULTRAPETALA1 and LEAFY pathways function independently in specifying identity and determinacy at the Arabidopsis floral meristem. Ann Bot. 2014;114(7):1497-505.

46. Nakamura Y, Andres F, Kanehara K, Liu YC, Dormann P, Coupland G. Arabidopsis florigen FT binds to diurnally oscillating phospholipids that accelerate flowering. Nat Commun. 2014:5:3553.

47. Chang J, Clay JM, Chang C. Association of cytochrome b5 with ETR1 ethylene receptor signaling through RTE1 in Arabidopsis. Plant J: cell and Mol Bio. 2014;77(4):558-67.

48. Wang Q, Zhang W, Yin Z, Wen CK. Rice CONSTITUTIVE TRIPLE-RESPONSE2 is involved in the ethylene-receptor signalling and regulation of various aspects of rice growth and development. J Exp Bot. 2013;64(16):4863-75.

49. Tsuchisaka A, Yu G, Jin H, Alonso JM, Ecker JR, Zhang X, et al. A combinatorial interplay among the 1-aminocyclopropane-1-carboxylate isoforms regulates ethylene biosynthesis in Arabidopsis thaliana. Genetics. 2009;183(3):979-1003.

50. Farcot $E$, Lavedrine $C$, Vernoux T. A modular analysis of the auxin signalling network. PLoS One. 2015;10(3):e0122231.

51. Ulmasov T, Murfett J, Hagen G, Guilfoyle TJ. Aux/IAA proteins repress expression of reporter genes containing natural and highly active synthetic auxin response elements. Plant Cell. 1997;9(11):1963-71.

52. Cho H, Ryu H, Rho S, Hill K, Smith S, Audenaert D, et al. A secreted peptide acts on BIN2-mediated phosphorylation of ARFs to potentiate auxin response during lateral root development. Nat Cell Biol. 2014;16(1):66-76.

53. Ellis CM, Nagpal P, Young JC, Hagen G, Guilfoyle TJ, Reed JW. Auxin response factor 1 and auxin response factor 2 regulate senescence and floral organ abscission in Arabidopsis thaliana. Development. 2005;132(20):4563-74.

54. Paull $\mathrm{R}$, Irikura B, Wu P, Turano $\mathrm{H}$, Chen N, Blas A, et al. Fruit development, ripening and quality related genes in the papaya genome. Tropical Plant Biol. 2008:1(3-4):246-77.

55. Ming R, Hou S, Feng Y, Yu Q, Dionne-Laporte A, Saw JH, et al. The draft genome of the transgenic tropical fruit tree papaya (Carica papaya Linnaeus). Nature. 2008:452(7190):991-6.

56. Young ND, Debelle F, Oldroyd GE, Geurts R, Cannon SB, Udvardi MK, et al. The Medicago genome provides insight into the evolution of rhizobial symbioses. Nature. 2011;480(7378):520-4.

57. Cole M, Chandler J, Weijers D, Jacobs B, Comelli P, Werr W. DORNROSCHEN is a direct target of the auxin response factor MONOPTEROS in the Arabidopsis embryo. Development. 2009;136(10):1643-51.

58. Li J, Dai X, Zhao Y. A role for auxin response factor 19 in auxin and ethylene signaling in Arabidopsis. Plant Physiol. 2006;140(3):899-908.

59. Wilmoth JC, Wang S, Tiwari SB, Joshi AD, Hagen G, Guilfoyle TJ, et al. NPH4/ ARF7 and ARF19 promote leaf expansion and auxin-induced lateral root formation. Plant J: cell and mol Bio. 2005;43(1):118-30.

60. Varaud E, Brioudes F, Szecsi J, Leroux J, Brown S, Perrot-Rechenmann C, et al. AUXIN RESPONSE FACTOR8 regulates Arabidopsis petal growth by interacting with the bHLH transcription factor BIGPETALp. Plant Cell. 2011;23(3):973-83.
61. Tabata R, Ikezaki M, Fujibe T, Aida M, Tian CE, Ueno Y, et al. Arabidopsis auxin response factor 6 and 8 regulate jasmonic acid biosynthesis and floral organ development via repression of class 1 KNOX genes. Plant Cell Physiol. 2010;51(1):164-75.

62. Hunter C, Willmann MR, Wu G, Yoshikawa M, de la Luz G-NM, Poethig SR. Trans-acting siRNA-mediated repression of ETTIN and ARF4 regulates heteroblasty in Arabidopsis. Development. 2006;133(15):2973-81.

63. Hayashi K. The interaction and integration of auxin signaling components. Plant Cell Physiol. 2012;53(6):965-75.

64. Uchiumi T, Okamoto T. Rice fruit development is associated with an increased IAA content in pollinated ovaries. Planta. 2010;232(3):579-92.

65. Gustafson FG. Inducement of fruit development by growth-promoting chemicals. Proc Natl Acad Sci U S A. 1936;22(11):628-36.

66. Wang $H$, Schauer $N$, Usadel B, Frasse P, Zouine M, Hernould M, et al. Regulatory features underlying pollination-dependent and -independent tomato fruit set revealed by transcript and primary metabolite profiling. Plant Cell. 2009;21(5):1428-52.

67. Schnable PS, Ware D, Fulton RS, Stein JC, Wei F, Pasternak S, et al. The B73 maize genome: complexity, diversity, and dynamics. Science. 2009;326(5956):1112-5.

68. de Jong M, Wolters-Arts M, Feron R, Mariani C, Vriezen WH. The Solanum lycopersicum auxin response factor 7 (SIARF7) regulates auxin signaling during tomato fruit set and development. Plant $\mathrm{J}$ : cell and Mol Bio. 2009;57(1):160-70.

69. Alexander L, Grierson D. Ethylene biosynthesis and action in tomato: a model for climacteric fruit ripening. J Exp Bot. 2002;53(377):2039-55.

70. Merchante C, Vallarino JG, Osorio S, Araguez I, Villarreal N, Ariza MT, et al. Ethylene is involved in strawberry fruit ripening in an organ-specific manner. J Exp Bot. 2013;64(14):4421-39.

71. Ruzicka K, Ljung K, Vanneste S, Podhorska R, Beeckman T, Friml J, et al. Ethylene regulates root growth through effects on auxin biosynthesis and transport-dependent auxin distribution. Plant Cell. 2007;19(7):2197-212.

72. Ivanchenko MG, Muday GK, Dubrovsky JG. Ethylene-auxin interactions regulate lateral root initiation and emergence in Arabidopsis thaliana. Plant J: cell and Mol Bio. 2008;55(2):335-47.

73. El-Sharkawy I, Sherif SM, Jones B, Mila I, Kumar PP, Bouzayen M, et al. TIR1-like auxin-receptors are involved in the regulation of plum fruit development. J Exp Bot. 2014;65(18):5205-15.

74. McMurchie EJ, McGlasson WB, Eaks IL. Treatment of fruit with propylene gives information about the biogenesis of ethylene. Nature. 1972;237(5352):235-6.

75. El-Sharkawy I, Sherif S, Mila I, Bouzayen M, Jayasankar S. Molecular characterization of seven genes encoding ethylene-responsive transcriptional factors during plum fruit development and ripening. J Exp Bot. 2009;60(3):907-22.

76. de Jong M, Wolters-Arts M, Garcia-Martinez JL, Mariani C, Vriezen WH. The Solanum lycopersicum AUXIN RESPONSE FACTOR 7 (SIARF7) mediates cross-talk between auxin and gibberellin signalling during tomato fruit set and development. J Exp Bot. 2011;62(2):617-26.

77. Liu KD, Li HL, Yuan CC, Huang YL, Chen Y, Liu JX. Identification of phenological growth stages of sugar apple (Annona squamosa L.) using the extended BBCH-scale. Sci Hortic-Amsterdam. 2015;181:76-80.

\section{Submit your next manuscript to BioMed Central and take full advantage of:}

- Convenient online submission

- Thorough peer review

- No space constraints or color figure charges

- Immediate publication on acceptance

- Inclusion in PubMed, CAS, Scopus and Google Scholar

- Research which is freely available for redistribution 